Related content

\section{Simultaneous readout of charge and scintillation pulses from electron avalanches for improving the response of micropattern gaseous detectors}

To cite this article: C M B Monteiro et al 2014 JINST 9 C08005

View the article online for updates and enhancements.
Micromegas operation in high pressure xenon: charge and scintillation readout C Balan, E D C Freitas, T Papaevangelou et al.
Secondary scintillation readout from GEM and THGEM with a large area avalanche photodiode
C M B Monteiro, L M P Fernandes, J F C A Veloso et al.
Simulation of VUV electroluminescence in micropattern gaseous detectors: the case of GEM and MHSP
C A B Oliveira, P M M Correia, H Schindler et al.

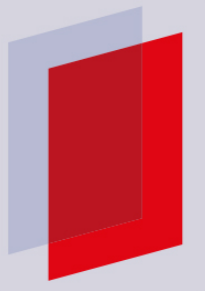

\section{IOP ebooks}

Bringing you innovative digital publishing with leading voices

to create your essential collection of books in STEM research.

Start exploring the collection - download the first chapter of every title for free. 


\title{
Simultaneous readout of charge and scintillation pulses from electron avalanches for improving the response of micropattern gaseous detectors
}

\author{
C.M.B. Monteiro, ${ }^{a, 1}$ C.A.B. Oliveira, ${ }^{b, c}$ H. Natal da Luz, ${ }^{a}$ J.F.C.A. Veloso ${ }^{a, b}$ and \\ J.M.F. dos Santos ${ }^{a}$ \\ ${ }^{a}$ GIAN, Physics Department, University of Coimbra, \\ 3004-516 Coimbra, Portugal \\ ${ }^{b}$ I3N - Physics Department, University of Aveiro, \\ 3810-193 Aveiro, Portugal \\ ${ }^{c}$ Lawrence Berkeley National Laboratory, \\ 1 Cyclotron Road, Berkeley, CA 94720, U.S.A. \\ E-mail: cristina@gian.fis.uc.pt
}

\begin{abstract}
Electroluminescence production and readout as amplification process for primary ionisation in time projection chambers (TPCs), are under study for application namely to rare event detection, where low-rate and high-radiation background are factors of ultimate importance, implying the highest achievable gain and best energy resolution in the detector. The use of the electroluminescence signal rather than the secondary charge signal provides these with additional advantages. However, the simplicity and low cost of charge readout electronics over scintillation readout systems imply that alternatives to electroluminescence production and readout as amplification process for primary ionisation are being investigated to apply to large scale detectors. The use of the secondary avalanche ionisation produced in micropattern electron multipliers like Large Electron Multipliers and also to gaseous detectors with pixelized readout for imaging is, therefore, investigated. Charge readout is simple and straightforward and is by far the most used readout method. A drawback is that the charge amplification method has energy resolutions that are typically worse than the scintillation amplification method, thus exploring methods to improve the energy resolution of the charge readout are always interesting.
\end{abstract}

\footnotetext{
${ }^{1}$ Corresponding author.
} 
In this work, we implement simultaneous readout of charge and scintillation produced in the avalanches of a Thick Electron Multiplier (THGEM) to explore the improvement of the detector response to ionising radiation in the charge readout channel. The charge readout could be used predominantly for position determination, while the scintillation readout would be used for energy measurement.

KEYWORDS: Charge transport, multiplication and electroluminescence in rare gases and liquids; Gaseous detectors; Micropattern gaseous detectors (MSGC, GEM, THGEM, RETHGEM, MHSP, MICROPIC, MICROMEGAS, InGrid, etc); Scintillators, scintillation and light emission processes (solid, gas and liquid scintillators) 


\section{Contents}

1 Introduction 1

2 Experimental setup and method 2

3 Experimental results and discussion $\quad 4$

4 Conclusions $\quad 7$

\section{Introduction}

Time projection chambers (TPCs) based on electroluminescence production and readout, as amplification process for primary ionisation are under investigation and/or implementation, namely for dark matter search [1,2] and neutrinoless double beta decay detection [3]. Given the characteristic low-rate, high-radiation background of these experiments it is crucial to have the highest possible gain and energy resolution in the detector. these can be achieved with the electroluminescence signal. Furthermore, in opposition to conventional electronic readout of secondary avalanche charge, the optical recording of the electroluminescence results in fast propagating pulses that are insensitive to electronic noise, RF pick-up and high-voltage issues, since the readout is physically and electrically decoupled from the gas-multiplication elements.

Nevertheless, alternatives to electroluminescence production and readout as the amplification process for primary ionisation are under investigation and/or implementation for large scale detectors, making use of the secondary avalanche ionisation produced in micropattern electron multipliers [4, 5]. Large Electron Multipliers (LEMs) are to be used in ArDM and the GLACIER programmes, and are an alternative to be considered for large scale upgrades of other experiments. The simplicity, low cost and much less mass burden of the charge readout system would be an important advantage over the scintillation readout with PMTs [1-3] or Si-PMs [3, 6, 7].

In addition, gaseous detectors with pixelized readout for imaging have been developed and applied for some time, now. Charge readout is a simple, straightforward method to collect and image the energy released in the detector, avoiding the complexity of a photosensor, and is by far the most used readout method. Yet, its performance is also, most of the times, worse than the scintillation amplification method, in terms of energy resolution, and means to improve that performance are always interesting paths to follow.

The light scintillation produced in charge avalanches has been investigated and its performance characteristics compared with the charge readout $[8-12,19]$. In this work, we perform simultaneous charge and scintillation readout of charge avalanches of GEMs to study the possibility of improving the detector response to ionising radiation in the charge readout channel. Pulse correlation is performed using the delay time between charge and scintillation pulses and using the relation between the amplitudes of both types of pulses. 


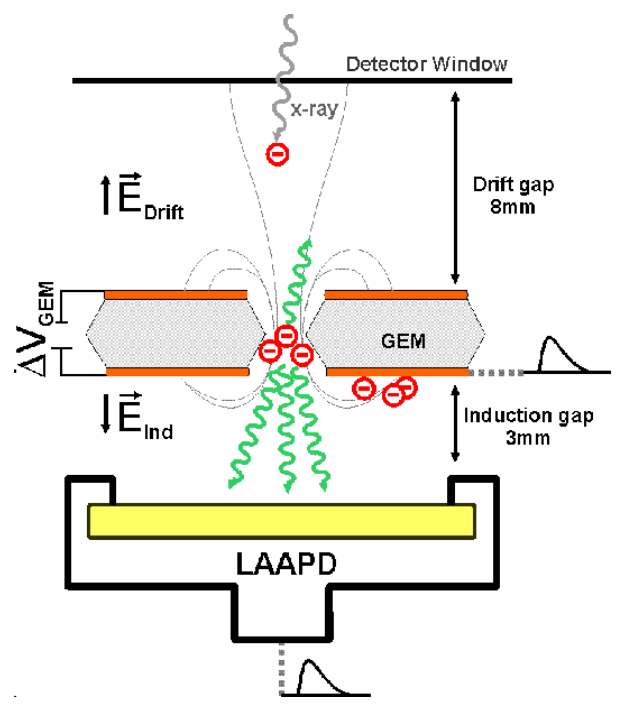

Figure 1. Gas Proportional Scintillation counter (GPSC) instrumented with a GEM and a Large Area Avalanche Photodiode as the photosensor. The charge avalanche was collected in the bottom electrode of the GEM.

\section{Experimental setup and method}

We have studied the avalanches produced in a micropattern gaseous detector equipped with a GEM for charge avalanche amplification of the ionizing radiation and with a Large Area Avalanche Photodiode (LAAPD) to readout the scintillation resulting from the avalanche processes. Figure 1 schematically depicts the GEM and the scintillation-readout LAAPD elements. This detector has already been used in $[10,11,13]$.

A stainless-steel chamber accommodated both GEM and LAAPD and was filled with 1 bar of pure xenon, after being pumped down to the $10^{-6}$ mbar region, being sealed off during the measurements. Gas purity was preserved by convection through non-evaporable getters (SAES St707), heated up to about $140^{\circ} \mathrm{C}$. The drift and induction gaps were 8 and $3 \mathrm{~mm}$ thick, respectively. The GEMs were manufactured at CERN and had standard dimensions, i.e. a $50 \mu \mathrm{m}$ Kapton foil with a $5 \mu \mathrm{m}$ copper clad on both sides and bi-conical holes of 50 and $70 \mu \mathrm{m}$ diameter in the Kapton and copper, respectively, arranged in a hexagonal layout of $140 \mu \mathrm{m}$ edges. The GEM active area was $2.8 \times 2.8 \mathrm{~cm}^{2}$. The LAAPD was an API Deep-UV sensitive model with an active diameter of $16 \mathrm{~mm}$.

A $1 \mathrm{~mm}$ diameter collimated $22.1 \mathrm{keV} \mathrm{X}$-ray beam interacting in the drift region induced primary electron clouds that were focused into the GEM holes, where they experienced charge avalanche multiplication. A reversed electric field was applied across the induction region for avalanche electrons to be collected on the bottom electrode (anode) of the GEM, see figure 1. A large number of VUV scintillation photons were produced in the charge avalanche as a result of the gas de-excitation processes. A fraction of these photons reached the LAAPD active-area and the corresponding electric signal was amplified in the photodiode.

LAAPD enclosure and chamber were at $0 \mathrm{~V}$; radiation window and GEM's top and bottom electrodes were biased independently. Constant drift- and induction-fields of 0.5 and $0.1 \mathrm{kV} / \mathrm{cm}$, 


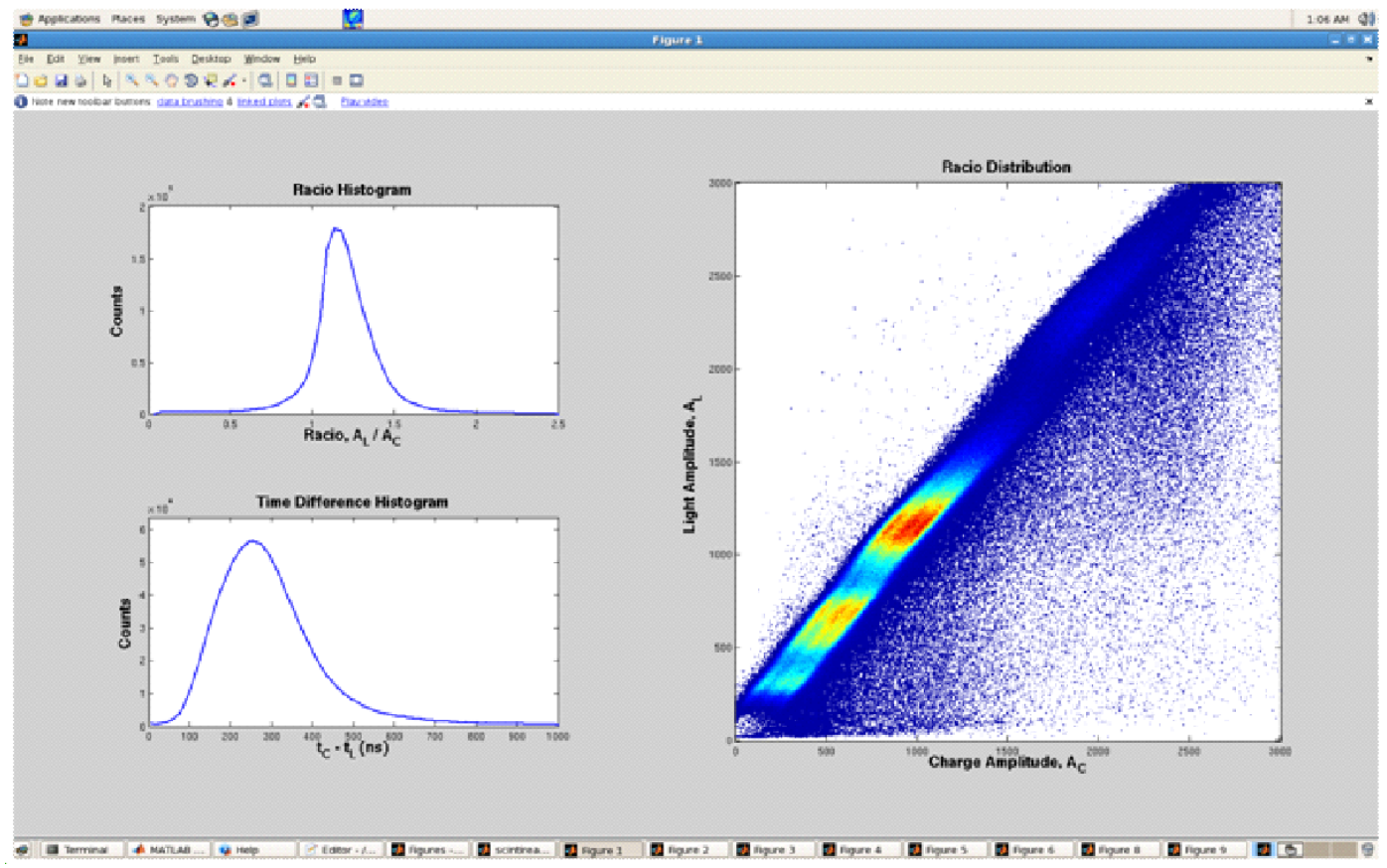

Figure 2. Scintillation and charge pulse correlation from a ${ }^{241} \mathrm{Am}$ radioactive source in a GEM operating in xenon at 1.0 bar, taken with the CAEN $1728 \mathrm{~b}$ board: amplitude ratio, time difference between two consecutive scintillation-charge pulses and amplitude-space parameter, i.e. pulse amplitude correlation plot.

respectively, were used throughout the measurements, as well as an LAAPD bias-voltage of $1840 \mathrm{~V}$, matching an LAAPD gain around 130 [14-16]. The charge avalanche gain and, thus, the electroluminescence gain were set by varying the voltage across the GEM's holes.

A CAEN $^{\mathrm{TM}}$ 1728b NIM module [17], equipped with 4 ADCs having a resolution of 14 bits and a $100 \mathrm{MHz}$ sampling rate was used to readout the charge pulses extracted from the bottom electrode of the GEM and the pulses obtained at the LAAPD output. The module performs realtime digital signal sampling and shaping, as well as data storage for offline analysis. It has a Xilinx Virtex II FPGA for pulse processing and hardware control and a Spartan II FPGA for system configuration [18]. For the purpose of this study, it was used in energy mode, wherein every event was stored up in a matrix with the pulse amplitude, time stamp, input channel and trigger number. All the parameters concerning data acquisition and processing can be adjusted according to the user needs. Each photon interacting in the gas provides two pulses, one from the scintillation and another from the charge readout channel, which are fed to two different channels of the modulePulses of both channels which are not appearing in a user defined coincidence window may be discarded. Figure 2 presents an example of typical parameters that can be displayed and selected to be used for pulse discrimination. These included the time difference between two consecutive pulses from the scintillation channel and the charge channel, within a $1 \mu$ s time window, the scintillation-to-charge pulse amplitude ratio, Asc/Ach, and the plot of the amplitudes of each pair of pulses, (Asc,Ach). This allowed obtaining pulse-height distributions for both scintillation and charge channels, with or without any discrimination constraints. 


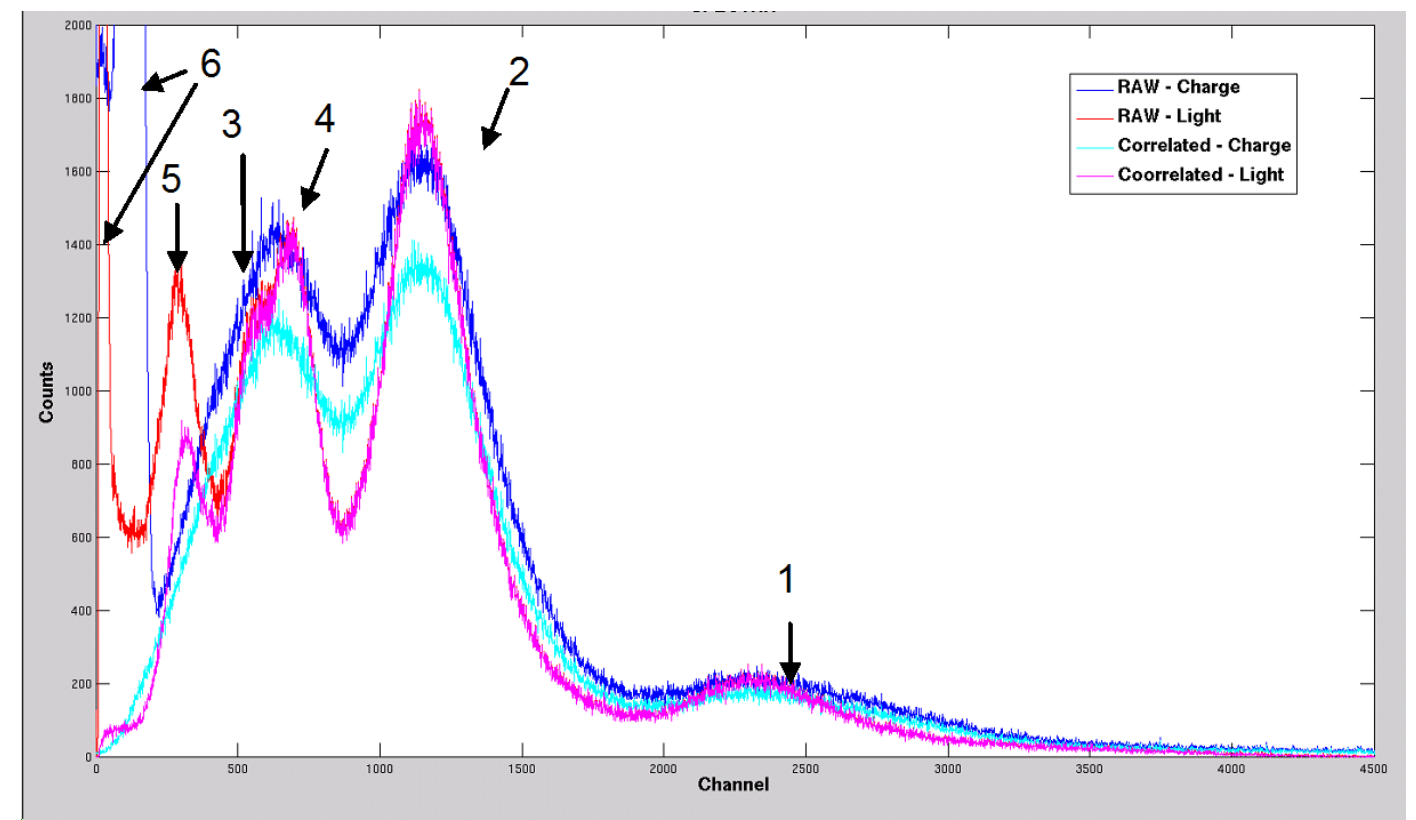

Figure 3. Scintillation and charge pulse height distributions for ${ }^{241} \mathrm{Am}$ photons obtained with a GEM operating in xenon, for the scintillation and charge channels and for two different cases: for no discrimination implemented (raw pulse-height distributions) and selecting only the pairs of pulses, one in each channel, that are within a microsecond (correlated pulse-height distributions). The raw pulse-height distributions exhibit the $59.6 \mathrm{keV}$ peak (1), the xenon K-fluorescence escape peak (2), the $\mathrm{Np}_{\alpha}$ - and $\mathrm{L}_{\beta}$-fluorescence emitted by the source $(3,4)$ and the electronic noise tail, in the low energy limit region $(6)$. The scintillation channel has also an additional peak due to direct X-ray interactions in the LAAPD (5).

\section{Experimental results and discussion}

Figure 3 depicts the pulse height distributions for ${ }^{241}$ Am photons obtained with a GEM operating in pure xenon, for both channels and for two different cases: no discrimination implemented (raw pulse height distributions) and selecting only the pairs of pulses, one in each channel, that appear within one microsecond (correlated pulse height distributions). The raw pulse height distributions exhibit the $59.6 \mathrm{keV}$ peak, the xenon K-fluorescence escape peak, the $\mathrm{Np} \mathrm{L} \alpha$ - and $\mathrm{L} \beta$-fluorescence emitted by the source and the electronic noise tail, in the low energy limit region. The scintillation channel has also an additional peak due to direct X-ray interactions in the LAAPD. The pulseheight distributions demonstrate the best performance by reading out the electroluminescence: improved energy resolution and reduced electronic noise.

Figure 3 shows that pulse correlation results in pulse-height distributions free of electronic noise and free of pulses that were only present in one of the channels, as are those resulting from direct X-ray interactions in the LAAPD. The small peak still present in the position of the LAAPD direct interactions corresponds to the $\mathrm{Np} \mathrm{L}$ fluorescence and/or events resulting from the xenon L-fluorescence escapes resulting from $\mathrm{Np} L \alpha$ - and $\mathrm{L} \beta$-fluorescence interactions in the xenon gas. This peak is not resolved (but noticeable) in the pulse-height distribution of the charge channel due to the poor energy resolution and it was superimposed on the peak resulting from direct X-ray absorptions in the LAAPD in the scintillation channel. 


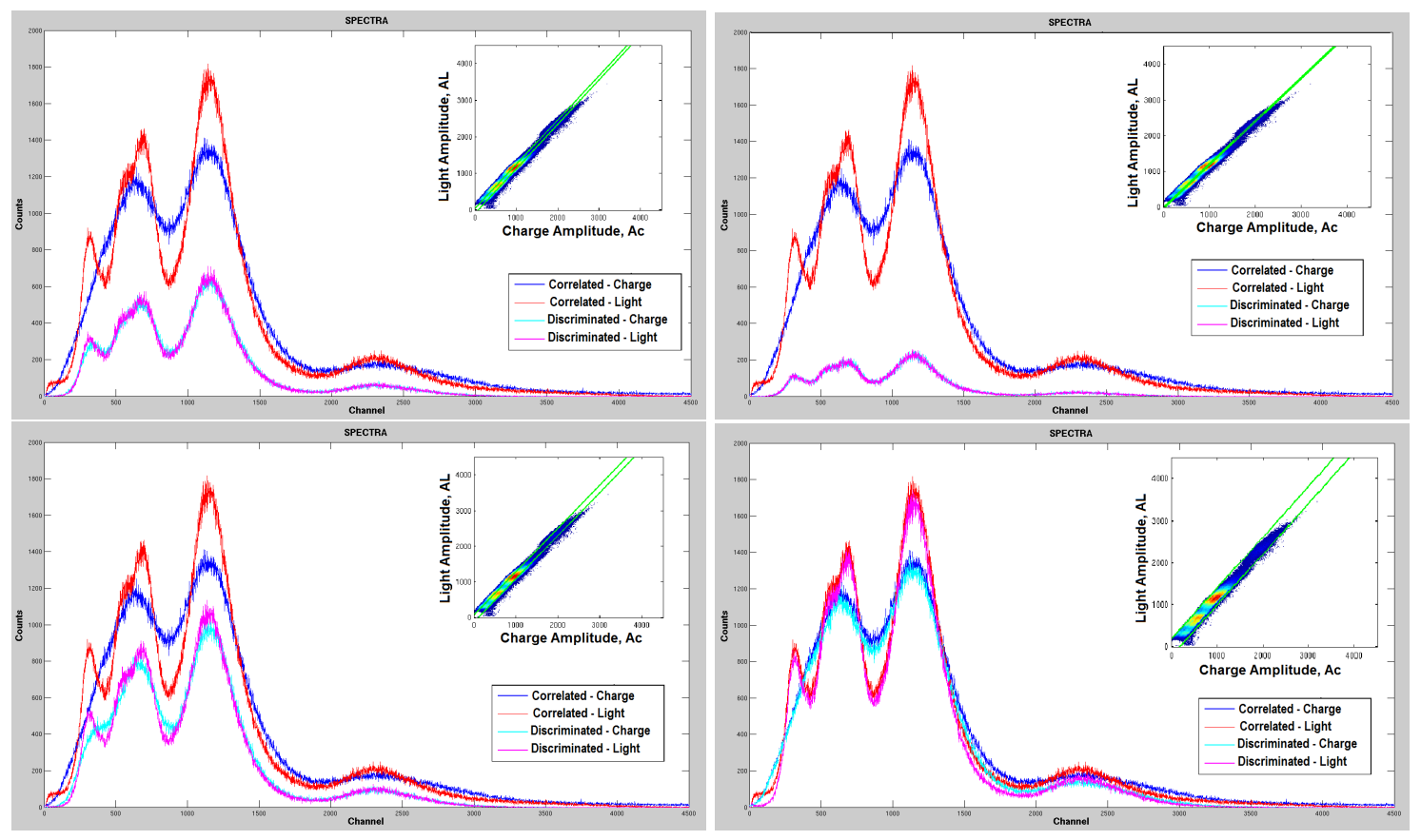

Figure 4. Scintillation and charge pulse-height distributions from ${ }^{241}$ Am photon interactions obtained with a GEM operating in xenon at $1.0 \mathrm{bar}$, using increasing areas of the pulse amplitude correlation plot for pulse discriminations, as indicated in each plot. For comparison, the total correlated pulse-height distributions, i.e. taking into account the whole area of the plot, was depicted in all the different graphs.

A more detailed discrimination can be performed, by making use of the scintillation and charge amplitude correlation plot, (Asc,Ach). As shown in figure 2, most of the pulse amplitudes were in a specific region. This is a consequence of the strong correlation that bonds both type of pulses, i.e. the scintillation pulse in the avalanche was produced by the secondary electrons meanwhile produced in the avalanche. Nevertheless, a small fraction of the pulses fell outside this area. In an offline analysis, we defined a small slab of the amplitude correlation plot and selected only the events that fell in that slab. However, the smaller the selected area for pulse discrimination, the lower the number of counts and pulse-height distribution statistics. Figure 4 presents different pulse-height distributions from ${ }^{241} \mathrm{Am}$ photon interactions using increasing areas of the pulse amplitude correlation plot for pulse discrimination, as indicated in each graph. For comparison, the total correlated pulse height distributions, i.e. taking into account the whole area of the amplitude correlation plot, was depicted in all the different graphs.

Figure 4 is intended to show that, if a narrow slab was chosen, the effective count-rate was low but the correlated charge and scintillation pulse-height distributions were similar, presenting the charge pulse distribution the same energy resolutions as those of the scintillation pulses. However, as the chosen slab widened, leading to an effective increase in the statistics, the energy resolution of the charge pulse-height distribution degraded.

Figure 5 depicts the ratio between the energy resolutions of the $30 \mathrm{keV} \mathrm{Xe} \mathrm{k} \alpha$ escape-peaks of the pulse-height distributions of the charge and scintillation pulses, corresponding to increasing larger slices of the amplitude-space parameter, as presented in figure 4. It is clear that the energy 


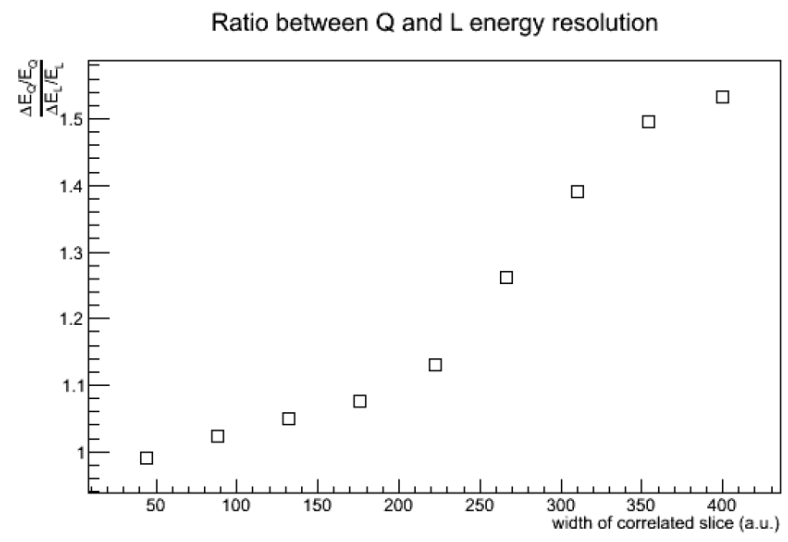

Figure 5. Ratio between the energy resolutions of the $30 \mathrm{keV} \mathrm{Xe} \mathrm{k} \alpha$ escape-peaks of the pulse-height distributions of the charge and scintillation pulses, corresponding to increasing larger slices of the amplitudespace parameter.

resolution became approximately the same when the slice was thinner, as expected. As the width of this slice increase, meaning that for a given light pulse amplitude, a larger range of charge pulse amplitudes was selected, the energy resolution of the charge channel degraded with respect to that of the scintillation light, up to a value that was $50 \%$ worse.

Although the energy performances of the charge channel are not sufficient, it would be interesting for imaging devices to use the charge collection to determine the event interaction position, while using the scintillation readout to perform event energy discrimination. Ideally, a single small area photosensor could be enough to implement such correction. However, in the real world, the area of coverage of the photosensor(s) is ruled by the need to read an enough amount of scintillation as to assure that the statistical fluctuations associated with the scintillation detection are close to optimum.

The time delay between consecutive scintillation and charge pulses as a possible pulse discrimination criterion was also studied. The scintillation pulse took place as the avalanche was developing, while the charge pulse took place after the drifting of the avalanche electrons out of the holes towards the anode electrode and the clearance of the positive ions out of the induction region. Figure 6a shows the distribution of the time difference between two consecutive pulses from the scintillation channel and the charge channel, respectively. We have divided this distribution in intervals, as depicted in figure 6a, and considered the pulse height distribution of the correlated pulses within these intervals. In figure $6 \mathrm{~b}$ we depict the energy resolution of the $30 \mathrm{keV} \mathrm{Xe} \mathrm{K \alpha}$ escape peak pulse-height distributions for the charge and scintillation pulses, corresponding to the different time-difference intervals of figure 6a. The dashed line is the energy resolution when all the events are considered. As shown, for some time-difference ranges, the energy resolution improved about $10 \%$. Although it has the cost of a reduction in statistics, the timing information may be an important tool for the study of the charge avalanche processes and charge collection, e.g. for an effective background reduction.

Energy resolution of the $30 \mathrm{keV} \mathrm{Xe} \mathrm{K} \alpha$ escape peak pulse-height distributions for the charge and scintillation pulses, corresponding to the different time-difference intervals of figure 6a. The dashed line is the energy resolution in the charge channel for all the events (right). 


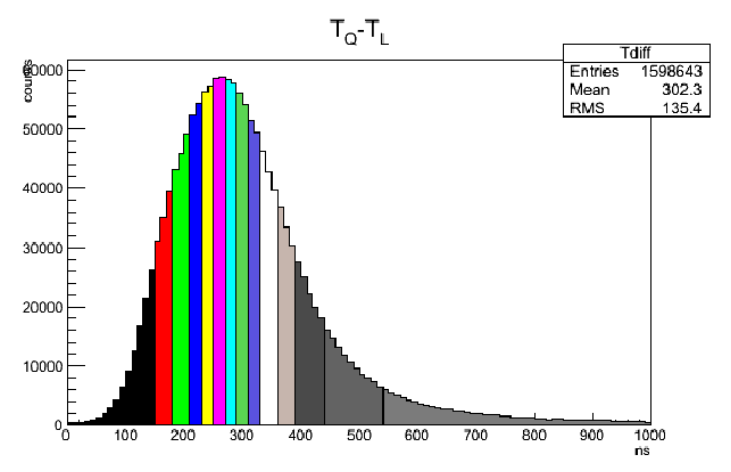

(a)

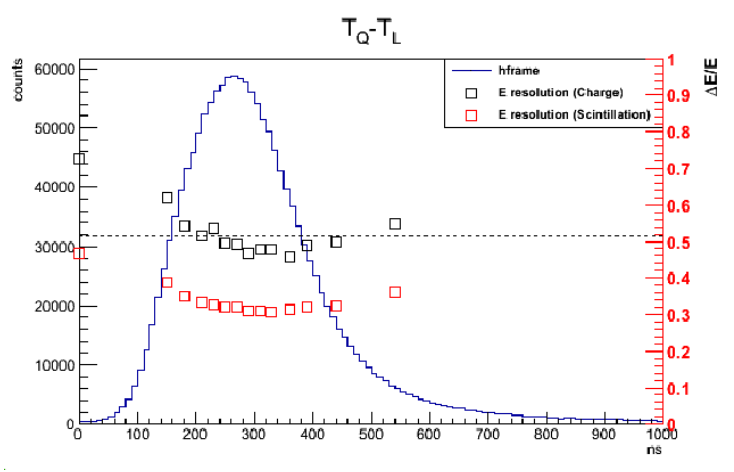

(b)

Figure 6. Distribution of the number of events as a function of the time difference between the pulses readout in the scintillation channel and in the charge channel (left).

\section{Conclusions}

We have investigated the correlation between the charge and the scintillation readout from avalanches produced in GEM holes and the possibility to improve the energy resolution that could be achieved in the charge readout channel using the information of the scintillation pulses. There was a strong correlation between the two types of pulses. Nevertheless, the statistical fluctuations associated with the scintillation detection were much less than those associated with the charge collection in the readout electrode, since the charge loss by diffusion and the charge division between the different electrodes will add additional fluctuations to the charge collection. On the other hand, an efficient reduction in the electronic noise could be achieved using coincidences between both pulses.

It is possible to improve the energy resolution associated to the charge readout using the pulse amplitude of the scintillation pulse as a discrimination parameter. However, this would be achieved at the cost of a significant reduction in the statistics and the use of the scintillation readout would be the solution to obtain the best energy resolution, without count rate reduction. Therefore, a good approach would be using the simplicity of charge readout channel for imaging and using the scintillation readout for energy discrimination. The use of the charge-pulse to scintillation-pulse time delay as a discrimination parameter did not allow a significant improvement in the energy resolution.

Nonetheless, the simultaneous readout of the charge and scintillation produced along the avalanches is an important tool for the study of the charge avalanche processes and charge collection in the readout electrodes, e.g. in MPGDs (Micro Pattern Gaseous Detector).

\section{Acknowledgments}

C.M.B. Monteiro is supported by FCT Grant SFRH/BPD/76842/2011. H. Natal da Luz is supported by FCT Grant SFRH/BPD/66737/2009. Work carried out within Project CERN/FP/123614/2011, under QREN initiative, UE/FEDER financing, through COMPETE. 


\section{References}

[1] XENON100 collaboration, E. Aprile et al., Dark Matter Results from 225 Live Days of XENON100 Data, Phys. Rev. Lett. 109 (2012) 181301 [arXiv:1207.5988].

[2] LUX collaboration, D.S. Akerib et al., First results from the LUX dark matter experiment at the Sanford Underground Research Facility, Phys. Rev. Lett. 112 (2014) 091303 [arXiv: 1310. 8214].

[3] NEXT collaboration, V. Alvarez et al., NEXT-100 Technical Design Report (TDR): Executive Summary, 2012 JINST 7 T06001 [arXiv: 1202.0721].

[4] A. Buzulutskov, Advances in Cryogenic Avalanche Detectors (review), 2012 JINST 7 C02025 [arXiv:1112.6153].

[5] A. Badertscher et al., First operation and performance of a 200 lt double phase LAr LEM-TPC with a $40 \times 76 \mathrm{~cm}^{2}$ readout, 2013 JINST 8 P04012 [arXiv:1301.4817].

[6] A. Bondar et al., Two-phase Cryogenic Avalanche Detectors with THGEM and hybrid THGEM/GEM multipliers operated in Ar and Ar+N2, 2013 JINST 8 P02008 [arXiv: 1210.0649].

[7] D.Y. Akimov et al., Two-phase xenon emission detector with electron multiplier and optical readout by multipixel avalanche Geiger photodiodes, 2013 JINST 8 P05017 [arXiv: 1303.7338].

[8] M.M.F.R. Fraga et al., The GEM scintillation in $\mathrm{He}-\mathrm{CF}_{4}, \mathrm{Ar}-\mathrm{CF}_{4}, \mathrm{Ar}-\mathrm{TEA}$ and Xe-TEA mixtures, Nucl. Instrum. Meth. A 504 (2003) 88.

[9] A. Bondar et al., On the low-temperature performances of THGEM and THGEM/G-APD multipliers in gaseous and two-phase Xe, 2011 JINST $6 \mathrm{P} 07008$ [arXiv: 1103.6126].

[10] C.M.B. Monteiro et al., Secondary scintillation yield from gaseous micropattern electron multipliers in direct dark matter detection, Phys. Lett. B 677 (2009) 133.

[11] C.M.B. Monteiro, L.M.P. Fernandes, J.F.C.A. Veloso, C.A.B. Oliveira and J.M.F. dos Santos, Secondary scintillation yield from GEM and THGEM gaseous electron multipliers for direct dark matter search, Phys. Lett. B 714 (2012) 18.

[12] A. Rubin et al., Optical readout: A tool for studying gas-avalanche processes, 2013 JINST 8 P08001 [arXiv:1305.1196].

[13] C.M.B. Monteiro, L.M.P. Fernandes, J.F. C.A. Veloso and J.M.F. dos Santos, Secondary scintillation readout from GEM and THGEM with a large area avalanche photodiode, 2012 JINST 7 P06012.

[14] L.M.P. Fernandes, J.A.M. Lopes, J.M.F. dos Santos and C.A.N. Conde, Application of large-area avalanche photodiodes to energy-dispersive $x$-ray fluorescence analysis, X-Ray Spectrom. 30 (2001) 164.

[15] J.A.M. Lopes, J.M.F. dos Santos, R.E. Morgado and C.A.N. Conde, A Xenon Gas Proportional Scintillation Counter with a UV-Sensitive Large-Area Avalanche Photodiode, IEEE Trans. Nucl. Sci. 48 (2001) 312.

[16] C.M.B. Monteiro et al., An Argon Gas Proportional Scintillation Counter With UV Avalanche Photodiode Scintillation Readout, IEEE Trans. Nucl. Sci. 48 (2001) 1081.

[17] CAEN, Via Vetraia, 11, 55049 - Viareggio (LU), Italy, Model N1728A/N1728B, 4 channel flash ADC, Revision $\mathrm{N}^{\circ}$ 5, October (2007).

[18] M. Ritcher and C. Santos, TNT2 digital pulse processor functionalities \& TUC control software, Centre National de la Recherche Scientifique, Institute Pluridisciplinaire Hubert Curien — Institute National de Physique Nucléaire et de Physique des Particules, Strasbourg, July (2007).

[19] J.M.F. dos Santos, Scintillation Readout From THGEMs operating in xenon, presented at $2^{\text {nd }}$ RD51 collaboration meeting, Paris, France, 13-15 October 2008, https://indico.cern.ch/event/35172/session/14/contribution/66/material/slides/1.pdf. 